Annals of Pure and Applied Mathematics

Vol. 21, No. 2, 2020, 113-118

ISSN: 2279-087X (P), 2279-0888(online)

Published on 2 May 2020

www.researchmathsci.org

DOI: http://dx.doi.org/10.22457/apam.v21n2a5646

Annals of

Pure and Applied

Mathematics

\title{
The $(a, b)$-Status Index of Graphs
}

\author{
V.R.Kulli
}

Department of Mathematics

Gulbarga University, Gulbarga 585 106, India

E-mail:vrkulli@gmail.com

Received 20 February 2020; accepted 21 April 2020

Abstract. The status of a vertex $u$ is defined as the sum of the distances between $u$ and all other vertices of a connected graph. In this paper, we introduce the $(a, b)$-status index of a graph. We also compute the $(a, b)$-status index of wheel and friendship graphs. Also we introduce $F_{1}$-status index, first and second status Gourava indices, symmetric division status index of a graph and compute exact formulas for wheel and friendship graphs.

Keywords: distance, $(a, b)$-status index, $F_{l}$-status index, symmetric division status index, graph.

\section{AMS Mathematics Subject Classification (2010): 05C05, $05 C 12$}

\section{Introduction}

Let $G$ be a finite, simple, connected graph. Let $V(G)$ be the vertex set and $E(G)$ be the edge set of $G$. The degree $d_{G}(u)$ of a vertex $u$ is the number of vertices adjacent to $u$. The distance, denoted by $d(u, v)$, between any two vertices $u$ and $v$ is the length of shortest path connecting $u$ and $v$. The status $\sigma(u)$ of a vertex $u$ in $G$ is the sum of distances of all other vertices from $u$ in $G$. For undefined terms and notations, we refer [1].

A graph index is a numerical parameter mathematically derived from the graph structure. The graph indices have their applications in various disciplines of Science and Technology [2,3]. Some of the graph indices can be found in $[4,5,6,7,8,9,10]$.

The first and second status connectivity indices were introduced by Ramane at al. in [11], defined as

$$
S_{1}(G)=\sum_{u v \in E(G)}[\sigma(u)+\sigma(v)], \quad S_{2}(G)=\sum_{u v \in E(G)} \sigma(u) \sigma(v) .
$$

In [12], Kulli introduced the product connectivity status index, defined as

$$
P S(G)=\sum_{u v \in E(G)} \frac{1}{\sqrt{\sigma(u) \sigma(v)}} .
$$

The reciprocal product connectivity status index and general second status index were introduced by Kulli in [12], and they are defined as

where $a$ is a real number.

$$
R P S(G)=\sum_{u v \in E(G)} \sqrt{\sigma(u) \sigma(v)} . \quad S_{2}^{a}(G)=\sum_{u v \in E(G)}[\sigma(u) \sigma(v)]^{a},
$$

We introduce the $F_{1}$-status index of a graph and is defined as 


$$
\begin{gathered}
\text { V.R.Kulli } \\
F_{1} S(G)=\sum_{u v E(G)}\left[\sigma(u)^{2}+\sigma(v)^{2}\right] .
\end{gathered}
$$

We introduce the first and second status Gourava indices of a graph, defined as

$$
\begin{gathered}
S G O_{1}(G)=\sum_{u v E(G)}[\sigma(u)+\sigma(v)+\sigma(u) \sigma(v)], \\
S G O_{2}(G)=\sum_{u v E E(G)} \sigma(u) \sigma(v)[\sigma(u)+\sigma(v)] .
\end{gathered}
$$

We define the symmetric division status index of a graph as

$$
\operatorname{SDS}(G)=\sum_{u v \in(G)}\left[\frac{\sigma(u)}{\sigma(v)}+\frac{\sigma(v)}{\sigma(u)}\right] .
$$

Motivated by the work on status indices, we introduce the $(a, b)$-status index of a graph and it is defined as

$$
S_{a, b}(G)=\sum_{u v E(G)}\left[\sigma(u)^{a} \sigma(v)^{b}+\sigma(u)^{b} \sigma(v)^{a}\right],
$$

where $a$ and $b$ are real numbers.

Recently the first and second Gourava indices were studied in [13].Recently, some status indices were introduced and studied such as multiplicative vertex status index [14], multiplicative first and second status indices [15], multiplicative $(a, b)$-status index [16], $F$-status index [17], $A B C$ status index [18], multiplicative $G A$ status index [19], harmonic status index [20], status connectivity coindices [21].In this paper, the (a, b)-status index of wheel and friendship graphs are determined.

\section{Observations}

We observe the following relationships.

1. The first status index $S_{1}(G)=S_{1,0}(G)$.

2. The second status index $S_{2}(G)=\frac{1}{2} S_{1,1}(G)$.

3. The product connectivity status index $P S(G)=\frac{1}{2} S_{-\frac{1}{2},-\frac{1}{2}}(G)$.

4. The reciprocal product connectivity status index $R P S(G)=\frac{1}{2} S_{\frac{1}{2}, \frac{1}{2}}(G)$.

5. The general second status index $S_{2}^{a}(G)=\frac{1}{2} S_{a, a}(G)$.

6. The $F_{1}$-status index $F_{1} S(G)=S_{2,0}(G)$.

7. The second status Gourava index $S G O_{2}(G)=S_{2,1}(G)$.

8. The symmetric division status index $\operatorname{SDS}(G)=S_{1,-1}(G)$.

\section{Results for wheel graphs}

A wheel graph $W_{n}$ is the join of $C_{n}$ and $K_{1}$. Then $W_{n}$ has $n+1$ vertices and $2 n$ edges. A graph $W_{n}$ is shown in Figure 1. 
The $(a, b)$-Status Index of Graphs

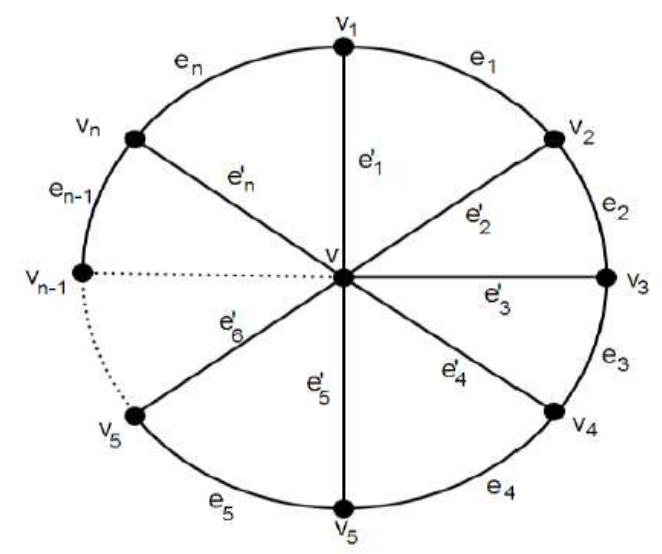

Figure 1: Wheel graph $W_{n}$

In $W_{n}$, there are two types of edges as follows:
$E_{1}=\left\{u v \in E\left(W_{n}\right) \mid d(u)=d(v)=3\right\}$,
$\left|E_{1}\right|=n$.
$E_{2}=\left\{u v \in E\left(W_{n}\right) \mid d(u)=3, d(v)=n\right\}$,
$\left|E_{2}\right|=n$.

Therefore there are two types of status edges as given in Table 1.
$\sigma(u), \sigma(v) \backslash u v \in E\left(W_{n}\right)$
$(2 n-3,2 n-3)$
$(n, 2 n-3)$

Number of edges

Table 1: Status edge partition of $W_{n}$

Theorem 1. The $(a, b)$-status index of a wheel graph $W_{n}$ is

$$
S_{a, b}\left(W_{n}\right)=n\left[2(2 n-3)^{a+b}\right]+n\left[n^{a}(2 n-3)^{b}+n^{b}(2 n-3)^{a}\right] .
$$

Proof : By using definition and Table 1, we deduce

$$
\begin{aligned}
& S_{a, b}\left(W_{n}\right)=\sum_{u v \in E\left(W_{n}\right)}\left[\sigma(u)^{a} \sigma(v)^{b}+\sigma(u)^{b} \sigma(v)^{a}\right] \\
& =n\left[(2 n-3)^{a}(2 n-3)^{b}+(2 n-3)^{b}(2 n-3)^{a}\right]+n\left[n^{a}(2 n-3)^{b}+n^{b}(2 n-3)^{a}\right] \\
& =n\left[2(2 n-3)^{a+b}\right]+n\left[n^{a}(2 n-3)^{b}+n^{b}(2 n-3)^{a}\right] .
\end{aligned}
$$

Corollary 1.1. From observations and by using equation (i), we establish the following results.
(1) $\quad S_{1}\left(W_{n}\right)=7 n^{2}-9 n$.
(2) $S_{2}\left(W_{n}\right)=6 n^{3}-15 n^{2}+9 n$.
(3) $\quad P S\left(W_{n}\right)=\frac{n}{2 n-3}+\frac{n}{\sqrt{n(2 n-3)}}$.
(4) $\quad R P S\left(W_{n}\right)=n(2 n-3)+n \sqrt{n(2 n-3)}$.
(5) $S_{2}^{a}\left(W_{n}\right)=(2 n-3)^{2 a} n+\left(2 n^{2}-3 n\right)^{a} n$.
(6) $\quad F_{1} S\left(W_{n}\right)=13 n^{3}-36 n^{2}+27 n$. 
(8) $\operatorname{SDS}\left(W_{n}\right)=\frac{9 n^{2}-18 n+9}{2 n-3}$.

Theorem 2. The first status Gourava index of a wheel graph $W_{n}$ is

$$
S G O_{1}\left(W_{n}\right)=6 n^{3}-8 n^{2}
$$

Proof: By definition, we have

$$
S G O_{1}\left(W_{n}\right)=\sum_{u v \in E\left(W_{n}\right)}[\sigma(u)+\sigma(v)+\sigma(u) \sigma(v)]
$$

Thus by using Table 1 , we deduce

$$
\begin{aligned}
S G O_{1} & \left(\mathrm{~W}_{n}\right)=n[(2 n-3)+(2 n-3)+(2 n-3)(2 n-3)] \\
& +n[n+(2 n-3)+n(2 n-3)] \\
& =6 n^{3}-8 n^{2} .
\end{aligned}
$$

\section{Results for friendship graphs}

A friendship graph $F_{n}, n \geq 2$, is a graph that can be constructed by joining $n$ copies of $C_{3}$ with a common vertex. A graph $F_{4}$ is presented in Figure 2.

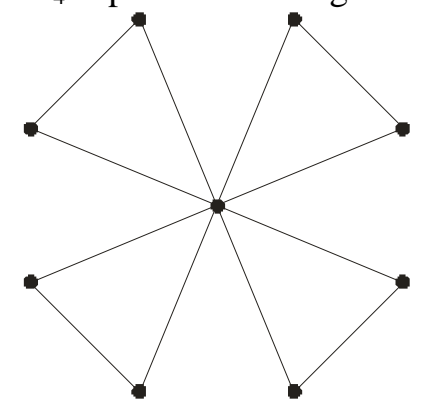

Figure 2: Friendship graph $F_{4}$

Let $F_{n}$ be a friendship graph with $2 n+1$ vertices and $3 n$ edges. By calculation, we obtain that there are two types of edges as follows:

$$
\begin{array}{ll}
E_{1}=\left\{u v \in E\left(F_{n}\right) \mid d_{F_{n}}(u)=d_{F_{n}}(v)=2\right\}, & \left|E_{1}\right|=n . \\
E_{2}=\left\{u v \in E\left(F_{n}\right) \mid d_{F_{n}}(u)=2, d_{F_{n}}(v)=2 n\right\}, & \left|E_{2}\right|=2 n .
\end{array}
$$

Therefore, in $F_{n}$, there are two types of status edges as given in Table 2.

$$
\sigma(u), \sigma(v) \backslash u v \in E\left(F_{n}\right) \quad(4 n-2,4 n-2) \quad(2 n, 4 n-2)
$$

Number of edges $n \quad 2 n$

Table 2: Status edge partition of $F_{n}$

Theorem 3. The $(a, b)$-status index of a friendship graph $F_{n}$ is given by

$$
S_{a, b}\left(F_{n}\right)=n\left[2(4 n-2)^{a+b}\right]+2 n\left[(2 n)^{a}(4 n-2)^{b}+(2 n)^{b}(4 n-2)^{a}\right] .
$$

Proof: From definition and by using Table 2, we obtain 
The $(a, b)$-Status Index of Graphs

$$
\begin{aligned}
& S_{a, b}\left(F_{n}\right)=\sum_{u v \in E\left(F_{n}\right)}\left[\sigma(u)^{a} \sigma(v)^{b}+\sigma(u)^{b} \sigma(v)^{a}\right] \\
& =n\left[(4 n-2)^{a}(4 n-2)^{b}+(4 n-2)^{b}(4 n-2)^{a}\right]+2 n\left[(2 n)^{a}(4 n-2)^{b}+(2 n)^{b}(4 n-2)^{a}\right] \\
& =n\left[2(4 n-2)^{a+b}\right]+2 n\left[(2 n)^{a}(4 n-2)^{b}+(2 n)^{b}(4 n-2)^{a}\right] .
\end{aligned}
$$

Corollary 3.1. From observations and by using equation (ii), we derive the following results.
(1) $S_{1}\left(F_{n}\right)=20 n^{2}-8 n$.
(2) $S_{2}\left(F_{n}\right)=32 n^{3}-24 n^{2}+4 n$.
(3) $\quad P S\left(F_{n}\right)=\frac{n}{4 n-2}+\frac{n}{\sqrt{n(2 n-1)}}$.
(4) $\operatorname{RPS}\left(F_{n}\right)=n(4 n-2)+4 n \sqrt{n(2 n-1)}$.
(5) $S_{2}^{a}\left(F_{n}\right)=(4 n-2)^{2 a} n+\left(8 n^{2}-4 n\right)^{a} 2 n$.
(6) $F_{1} S\left(F_{n}\right)=72 n^{3}-64 n^{2}+16 n$.
(7) $S_{G O}\left(F_{n}\right)=2 n(4 n-2)\left(12 n^{2}-2\right)$.
(8) $\operatorname{SDS}\left(F_{n}\right)=\frac{14 n^{2}-10 n+2}{2 n-1}$.

Theorem 4. The first status Gourava index of a friendship graph $F_{n}$ is

$$
S G O_{1}\left(F_{n}\right)=32 n^{3}-4 n^{2}-4 n \text {. }
$$

Proof: By definition, we have

$$
S G O_{1}(G)=\sum_{u v \in E(G)}[\sigma(u)+\sigma(v)+\sigma(u) \sigma(v)]
$$

Thus by using Table 2, we obtain

\section{Conclusion}

$$
\begin{aligned}
S G O_{1}\left(F_{n}\right) & =[(4 n-2)+(4 n-2)+(4 n-2)(4 n-2)] n \\
& +[2 n+(4 n-2)+2 n(4 n-2)] 2 n \\
& =32 n^{3}-4 n^{2}-4 n .
\end{aligned}
$$

In this study, the $(a, b)$-status index and some other status indices for particular values of $a$ and $b$ for wheel graphs and friendship graphs are computed.

Acknowledgement. The author is thankful to the referee for useful suggestions.

\section{REFERENCES}

1. V.R.Kulli, College Graph Theory, Vishwa International Publications, Gulbarga, India (2012).

2. V.R.Kulli, Multiplicative Connectivity Indices of Nanostructures, LAP LEMBERT Academic Publishing, (2018).

3. R.Todeschini and V.Consonni, Handbook of Molecular Descriptors for Chemoinformatics, Wiley-VCH, Weinheim, (2009). 
4. B.Basavanagoud and P.Jakkannavar, Kulli-Basava indices of graphs, Inter. J. Appl. Eng. Research, 14(1) (2018) 325-342.

5. S.Ediz, Maximal graphs of the first reverse Zagreb beta index, TWMS J. Appl. Eng. Math., 8 (2018) 306-310.

6. V.R.Kulli, On $K V$ indices and their polynomials of two families of dendrimers, International Journal of Current Research in Life Sciences, 7(9) (2018) 2739-2744.

7. V.R.Kulli, Dakshayani indices, Annals of Pure and Applied Mathematics, 18(2) (2018) 139-146.

8. V.R.Kulli, Computation of some temperature indices of $H C 5 C 7[p, q]$ nanotubes, Annals of Pure and Applied Mathematics, 20(2) (2019) 69-74.

9. V.RKulli, The $(a, b)$-temperature index of $H$-Naphtalenic nanotubes, Annals of Pure and Applied Mathematics, 20(2) (2019) 85-90.

10. I.Gutman, V.R.Kulli, B.Chaluvaraju and H.S.Boregowda, On Banhatti and Zagreb indices, Journal of the International Mathematical Virtual Institute, 7 (2017) 53-67.

11. H.S.Ramane and A.S.Yalnaik, Status connectivity indices of graphs and its applications to the boiling point of benzenoid hydrocarbons, Journal of Applied Mathematics and Computing, 55 (2017) 609-627.

12. V.R.Kulli, Some new status indices of graphs, International Journal of Mathematics Trends and Technology, 65(10) (2019) 70-76.

13. V.R.Kulli, The Gourava indices and coindices of graphs, Annals of Pure and Applied Mathematics, 14(1) (2017) 33-38.

14. V.R.Kulli, Computation of multiplicative status indices of graphs, International Journal of Mathematical Archive, 11(4) (2020) 1-6.

15. V.R.Kulli, Some new multiplicative status indices of graphs, International Journal of Recent Scientific Research, 10(10) (2019) 35568-35573.

16. V.R.Kulli, Computation of multiplicative $(a, b)$-status index of certain graphs, Journal of Mathematics and Informatics, 18 (2020) 45-50.

17. V.R.Kulli, Computation of status indices of graphs, International Journal of Mathematics Trends and Technology, 65(12) (2019) 54-61.

18. V.R.Kulli, Computation of $A B C, A G$ and augmented status indices of graphs, International Journal of Mathematics Trends and Technology, 66(1) (2020) 1-7.

19. V.R.Kulli, Multiplicative $A B C, G A, A G$, augmented and harmonic status indices of graphs, International Journal of Mathematical Archive, 11(1) (2020) 32-40.

20. H.S.Ramane, B.Basavanagoud and A.S.Yalnaik, Harmonic status index of graphs, Bulletin of Mathematical Sciences and Applications, 17(2016) 24-32.

21. H.S.Ramane, A.S.Yalnaik and R.Sharafdini, Status connectivity indices and coindices of graphs and its computation to some distance balanced graphs, AKCE International Journal of Graphs and Combinatorics, (2018) https://doi.org/10.1016j.2018.09.002. 